\title{
Depressione, stile psicotico e bassa autostima nell'ideazione e nei comportamenti suicidari
}

\author{
Roberto Infrasca ${ }^{1}$
}

1 Servizio di Salute Mentale, U.O. di Psichiatria ASL5, La Spezia

\begin{abstract}
Psychiatric disorders are the most important risk factor for suicidal behaviour. Many studies have shown that the suicide plays a crucial role in the structuring of self-injurious behaviours implemented to psychiatric patients. Numerous researchers reported that depressive symptoms, low self-esteem, and "psychotic style or psychoticism", are the most important conditions involved in suicidal ideation and suicidal behaviour. In this area a significant aspect of clinical interest is the suicidal ideation. The present study aimed to evaluate the prevalence of suicidal ideation among a sample of psychiatric patients, and in general population. The total sample consisted of 1,130 females (mean age $=34.9$; $\mathrm{sd}=13.8$ ) and 686 males (mean age $=38.6$; $\mathrm{sd}=13.5$ ). The study sample included anxiety disorders - AD ( $\mathrm{n}=493 ; 27.1 \%)$, depressive disorders - DD $(\mathrm{n}=768 ; 42.3 \%)$, somatoform disorders $-\mathrm{sd}(\mathrm{n}=$ $370 ; 20.4 \%)$, and general population - GP $(\mathrm{n}=185 ; 10.2 \%)$. All subjects were required to complete the MMPI test, including the MMPI scales for depression (D), schizophrenia (Sc), and Low Self-Esteem (LSE). Two indices were also obtained from the MMPI to measure suicidal ideation: the Threat Suicide (TS) scale, and the response to the item 339 («Most of the time I wish I was dead»: true $=1$; false = 0 ). Each subject of the sample is characterised by a personal clinical profile organised by the absence/presence of the three scales (normal or pathological), together with positivity or negativity of the two indices. The statistical analysis were performed by logistic regression (LR), analysis of variance, Chi-square $\left(\chi^{2}\right)$, and linear regression (Pearson's r). Data emerging from this study support the validity of three scales used for assessment of suicidal risk. The results also showed the strong relationship between depression, suicidal ideation, and suicidal behaviour, compared to the other analysed groups, together with less suicidal risk highlighted by the general population.
\end{abstract}

Keywords: depression, psychotic style, low self-esteem, suicidal ideation, suicidal behaviour

Depression, psychotic style and low self-esteem in suicidal ideation and suicidal behaviour

Pratica Medica \& Aspetti Legali 2010; 4(2): 57-62

\section{INTRODUZIONE}

Le patologie psichiatriche costituiscono il più importante fattore di rischio per i comportamenti suicidari. Una ricerca ha evidenziato che in un campione di 5.412 pazienti psichiatrici ospedalizzati il rischio suicidario era da 11 a 67 volte più elevato rispetto alla popolazione generale [1].

La previsione e/o prevenzione dei comportamenti suicidari rappresenta uno degli aspetti più difficili incontrati sia dalla pratica clinica sia dalla ricerca [2], non essendo ancora stati individuati segni clinici specifici volti a evidenziare il rischio di tali comportamenti [3]. Alcuni Autori valutano che circa il $10 \%$ dei pazienti depressi adulti ha messo in atto un tentativo di suicidio [4,5], comportamento autolesivo che riguarda l' $8-10 \%$ dei maschi, e il 20 $26 \%$ delle femmine [6].

In questo specifico ambito un aspetto di rilevante interesse clinico si evidenzia nell'ideazione suicidaria, costrutto mentale che si organizza intorno a idee, fantasie e vissuti che stimolano nel soggetto 
una percezione di "positività" nei confronti della morte. Numerosi studi sostengono che tale condizione "ideativa" gioca un ruolo essenziale nella strutturazione dei comportamenti autolesivi agiti dai pazienti psichiatrici [7-10].

In letteratura numerose ricerche hanno individuato nella sintomatologia depressiva [10-12], nella bassa autostima [13-15], nello "stile psicotico o psicoticismo" [16,17], le condizioni maggiormente implicate nelle dinamiche suicidarie, sia nella loro manifestazione mentale (ideazione), sia comportamentale (tentato suicidio e suicidio completato). Tali variabili cliniche, peraltro, possono essere agevolmente misurate attraverso il MMPI (scale fondamentali, scale speciali e indici) [18].

La sintomatologia depressiva si esprime attraverso l'abbassamento del tono dell'umore, la presenza di idee pessimistiche, di sentimenti di inadeguatezza e di colpa, di disperazione, la perdita di interesse, l'apatia, la negazione di esperienze piacevoli e la riduzione delle capacità lavorative. Quadri depressivi gravi si manifestano tramite modalità di reazione di tipo depressivo in situazioni stressogene, scarsa fiducia nelle proprie capacità, senso di inutilità e di angoscia, tratti introversivi e viraggi disforici. Inoltre, si rileva la presenza di pensieri pessimistici, di sentimenti malinconici, di vuoto, di disperazione, di incertezza sul futuro, una generale indifferenza verso la vita, la possibile presenza di idee di morte e suicidarie, sentimenti di colpa e bassa energia vitale. Lo stile psicotico o psicoticismo è riferito al distacco e alla chiusura sociale. Lo psicoticismo viene ritrovato in personalità che hanno uno scarso contatto con la realtà, riferiscono esperienze insolite o manifestano comportamenti bizzarri (nei casi più gravi deliri e allucinazioni). Questi soggetti tendono ad avere uno stile di vita schizoide, avvertono un basso senso di appartenenza al contesto sociale, sono isolati, si estraniano facilmente, hanno difficoltà a differenziare il sogno dalla realtà. Inoltre, risultano egocentrici, non convenzionali, manifestano preoccupazioni di tipo sessuale, stato d'ansia generalizzato, perdita del controllo dell'lo, mancanza di inibizioni, esperienze sensoriali bizzarre. L'elevazione del tasso di psicoticismo indica così la potenziale disposizione a sviluppare sintomi psichiatrici, pur se non necessariamente in forma di psicosi.

La stima di sé riguarda i sentimenti del soggetto nei confronti di se stesso, il modo in cui si vede, si giudica e si attribuisce valore. L'alta o la bassa autostima si riflettono su tre importanti aspetti della vita: il proprio modo di presentarsi agli altri, il passare dal pensiero all'azione per poter realizzare $\mathrm{i}$ propri obiettivi, e infine il modo di reagire a successi e insuccessi. Una bassa autostima viene rintracciata in soggetti con autopercezione negativa, tendenza ad atteggiamenti autosvalutativi e sfavorevoli nei confronti di se stessi, difficoltà nell'accogliere affermazioni di stima o approvazione, pen- sieri di essere poco attraenti, goffi, incapaci, inutili e di peso per gli altri. Baumgardner afferma che le persone con bassa autostima impiegano solitamente più tempo degli altri nel parlare di sé, a volte lo fanno con un certo imbarazzo e non sempre riescono convincenti quando si tratta di descrivere qualità di fatto possedute [19].

Branden ritiene che i soggetti con una bassa autostima manifestano una "felicità ansiosa", che prevede - in caso di successo - la difficoltà ad assaporare il risultato raggiunto, conseguente alla percezione della sua temporaneità, e al dover affrontare imminenti delusioni (date per scontate) [20].

In questa prospettiva, le idee suicidarie e i propositi suicidi assumono così un profilo variegato: evidenziano la loro profonda delicatezza, mettono in luce un'organizzazione multidimensionale, dimostrano un aspetto prevalentemente anonimo e silente. A questo ultimo riguardo, alcuni studi hanno verificato che solo il 3-5\% delle persone che minacciano un suicidio esprime in maniera evidente e inequivocabile l'intenzionalità suicidaria [21]. Nonostante questa ultima difficoltà, una ricerca svolta su pazienti afferenti a strutture di medicina generale, ha accertato che - ponendo ai soggetti domande relative ai disturbi del sonno, al calo del tono dell'umore, ai sensi di colpa e alla disperazione - è possibile identificare correttamente l'84\% dei soggetti che ha avuto pensieri suicidari nel corso dell'anno precedente [22]. Infine, altri studi hanno verificato che il tentativo di suicidio era fortemente associato al numero di disturbi psichiatrici, mentre l'ideazione suicidaria risultava maggiormente correlata agli abusi fisici subiti nell'infanzia [23]. Sulla scorta delle considerazioni svolte, lo studio qui descritto si propone di verificare un modello statistico predittivo del rischio suicidario nel paziente psichiatrico, basato sulle variabili cliniche ribadite in letteratura e in alcune ricerche sull'argomento [24], unitamente all'analisi degli effetti di interazione tra le stesse.

\section{METODI}

Il campione analizzato era formato da 1.130 femmine (età media $=34,9 ; \mathrm{sd}=13,8$ ) e 686 maschi (età media $=38,6$; $\mathrm{sd}=13,5$ ), e risultava così suddiviso:

- disturbi d'ansia (DA): $\mathrm{n}=493 ; 27,1 \%$;

- disturbi depressivi (DD): $\mathrm{n}=768 ; 42,3 \%$;

- disturbi somatoformi (DS): $\mathrm{n}=370 ; 20,4 \%$.

Al campione è inoltre stato affiancato un gruppo di controllo reperito dalla popolazione generale (PG): $\mathrm{n}=185 ; 10,2 \%$.

Le diagnosi sono state formulate in accordo con $\mathrm{i}$ criteri del DSM IV. 
Al campione è stato somministrato il MMPI (Minnesota Multiphasic Personality Inventory) dal quale sono state ricavate le tradizionali scale cliniche, unitamente alla scala LSE (Low Self-Esteem). Dal MMPI sono stati ottenuti anche due indicatori suicidari che valutano rispettivamente la "minaccia di suicidio" (scala Threat Suicide) [25], e la presenza di ideazione suicidaria (risposta "vero" all'item 339: "Quasi sempre vorrei essere morto»), utilizzati per le analisi statistiche.

Per il modello predittivo sono state adottate le scale cliniche D (depressione), Sc (schizofrenia) e la scala LSE (Low Self-Esteem). Il punteggio di ogni scala è stato anche sottoposto a una codificazione binaria, che assume i valori 0 (valore numerico non patologico della scala) o 1 (valore numerico patologico della scala). Ogni soggetto del campione è quindi caratterizzato da un personale profilo clinico organizzato dalla assenza/ presenza (valore normale o patologico) delle tre scale, unitamente alla positività (1) o negatività (0) dei due indicatori menzionati. Le analisi statistiche sono state effettuate attraverso la regressione logistica (RL), l'analisi della varianza, il Chiquadrato $\left(\chi^{2}\right)$, e la regressione lineare ( $r$ di Pearson).

\section{RISULTATI}

Nel campione il $14,1 \%(n=256)$ dei soggetti presenta ideazione suicidaria: il 17,6\% delle femmine ( $n=199)$, e l'8,3\% dei maschi $(n=57)$. Nei gruppi indagati tale indicatore si distribuisce nel modo seguente:

- disturbi d'ansia: 30,5\%;

- disturbi depressivi: 56,3\%;

- disturbi somatoformi: 12,5\%;

- popolazione generale: $0,8 \%$; con $\chi^{2}=49,9 ; \mathrm{df}=3 ; \mathrm{p}=0,000000$.

\section{SIGLE UTILIZZATE}

DA: disturbi d'ansia

DD: disturbi depressivi

df: degrees of freedom

DS: disturbi somatoformi

DSM IV: Diagnostic and Statistic Manual of mental disorders, Fourth Edition

F: test-value

IC: intervallo di confidenza

MMPI test: Minnesota Multiphasic Personality Inventory

p: p-value

PG: popolazione generale

OR: Odds Ratio

RL: regressione logistica

r: regressione lineare

Scala LSE: scala Low Self-Esteem

Scala D: scala depressione

Scala Sc: scala schizofrenia

Scala TS: scala Threat Suicide

sd: standard deviation

\begin{tabular}{|cc|cc|cc|}
\multicolumn{2}{l}{ DS } & \multicolumn{2}{c}{ DA } & \multicolumn{2}{c}{ DD } \\
\hline OR & IC $95 \%$ & OR & IC 95\% & OR & IC $95 \%$ \\
\hline 8,6 & $2,0-36,5$ & 17,1 & $4,1-70,7$ & 21,1 & $5,1-86,0$ \\
\hline
\end{tabular}

Tabella I. Ogni quadro clinico presenta un diverso rischio di ideazione suicidaria rispetto alla popolazione generale

$\mathrm{DA}=$ disturbi d'ansia; DD = disturbi depressivi; DS = disturb somatoformi; IC = intervallo di confidenza; OR =Odds Ratio

Rispetto alla PG, la regressione logistica ha messo in luce che il rischio di ideazione suicidaria risulta differenziato rispetto al quadro clinico (Tabella I): $\mathrm{DS}(\mathrm{OR}=8,6)$; $\mathrm{DA}(\mathrm{OR}=17,1)$ o $\mathrm{DD}(\mathrm{OR}=21,1)$. Una verifica statistica di quanto affermato è stata ottenuta attraverso la RL tra la positività della scala Threat Suicide $(0 \leq 70 ; 1>70$ punti) del MMPI nei diversi gruppi. Di fatto, rispetto alla PG, la "mi-

\begin{tabular}{|lcc|ccc|c|c|} 
& \multicolumn{3}{c}{ DS } & \multicolumn{2}{c}{ DA } & DD \\
\cline { 2 - 7 } & 个risp PG & IC $95 \%$ & $\uparrow$ risp PG & IC $95 \%$ & $\uparrow$ risp PG & IC 95\% \\
\hline Minaccia di suicidio & 11,5 & $4,1-31,9$ & 22,5 & $8,2-61,8$ & 31,9 & $11,7-87,0$ \\
\hline $\begin{array}{l}\text { Scala Sc: rischio di presentare uno stile } \\
\text { psicotico }\end{array}$ & 17,3 & $6,9-43,4$ & 30,7 & $12,4-76,2$ & 38,3 & $15,5-94,1$ \\
\hline Scala LSE: rischio di bassa autostima & 29,6 & $10,7-81,6$ & 68,1 & $24,9-186,6$ & 123,5 & $45,2-337,1$ \\
\hline $\begin{array}{l}\text { Scala D: rischio di presenza di una } \\
\text { struttura depressiva }\end{array}$ & 19,0 & $10,4-34,7$ & 22,8 & $12,6-41,3$ & 100,3 & $54,7-183,8$ \\
\hline
\end{tabular}

Tabella II. Misurazione delle variabili con le diverse scale sui tre tipi di quadri clinici, espresse come aumenti in numero di volte rispetto alla popolazione generale (PG)

$D A$ = disturbi d'ansia; $D D=$ disturbi depressivi; $D S=$ disturbi somatoformi; $I C=$ intervallo di confidenza 
naccia di suicidio" è 11,5 volte più elevata nei $\mathrm{DS}$, 22,5 nei DA e 31,9 nei DD.

Si nota agevolmente come i soggetti affetti da disturbi depressivi mostrino un maggiore rischio di ideazione suicidaria (2,4 volte rispetto ai DS, 0,8 ai DA), e di potenziale comportamento suicidario $(2,8$ volte rispetto ai DS, e 0,7 ai DA) in relazione agli altri due gruppi psichiatrici. Tale risultato ha trovato una giustificazione nella maggiore disorganizzazione psicopatologica della condizione depressiva rispetto alle altre. La regressione logistica ha evidenziato che il rischio che la personalità presenti uno stile psicotico (scala Sc MMPI) risulta 17,3 volte più elevato nei DS, 30,7 nei DA e 38,3 nei DD. La probabilità di bassa autostima (scala LSE) non segue un destino diverso: è 29,6 volte più elevata nei $\mathrm{DS}, 68,1$ nei DA e 123,5 nei DD. La positività della scala D del MMPI, unitamente ai tratti psicopatologici ad essa correlati, mostra un andamento ancora più significativo. Il rischio della presenza di una struttura depressiva della personalità è 19,0 volte per i soggetti con DS, 22,8 per i DA e 100,3 per i DD (Tabella II).

I risultati ottenuti portano a sostenere che la tipologia di interazione tra le scale Sc, D e LSE del MMPI (nessuna scala positiva presente, oppure presenza di una, due o tre scale positive) e i loro

\begin{tabular}{|lcc|}
\multicolumn{1}{r}{ Scale } & OR & IC $95 \%$ \\
\hline D & 2,5 & $1,0-6,1$ \\
\hline LSE-D & 5,3 & $2,5-11,0$ \\
\hline Sc-D & 6,0 & $1,2-29,5$ \\
\hline Sc & 9,2 & $3,4-24,6$ \\
\hline Sc-LSE & 13,9 & $3,3-57,6$ \\
\hline Sc-LSE-D & 20,5 & $10,7-39,2$ \\
\hline
\end{tabular}

Tabella III. II rischio di ideazione suicidaria rilevato da una scala o da combinazioni di scale, ottenuto con regressione logistica tra ideazione suicidaria e comportamento suicida misurati con le diverse scale

$\mathrm{D}=$ scala depressione; $\mathrm{IC}=$ intervallo di confidenza; $\mathrm{LSE}=$ scala Low Self-Esteem; OR = Odds Ratio; $\mathrm{Sc}=$ scala schizofrenia

\begin{tabular}{|lcc|}
\multicolumn{1}{r}{ Scale } & OR & IC $95 \%$ \\
\hline D & 2,9 & $1,3-6,3$ \\
\hline Sc & 9,2 & $3,8-22,4$ \\
\hline Sc-D & 13,9 & $4,3-44,4$ \\
\hline LSE-D & 16,3 & $8,8-29,2$ \\
\hline Sc-LSE-D & 62,8 & $35,4-111,4$ \\
\hline Sc-LSE & 70,2 & $20,6-238,7$ \\
\hline
\end{tabular}

Tabella IV. Aumento del rischio suicidario rilevato da una scala o da combinazioni di scale, ottenuto con regressione logistica tra la scala Threat Suicide e le diverse combinazioni assunte dalle scale analizzate

$D$ = scala depressione; $I C$ = intervallo di confidenza; $L S E$ = scala Low Self-Esteem; OR = Odds Ratio; $\mathrm{Sc}=$ scala schizofrenia significati psicopatologici, possono diminuire o aumentare il rischio di ideazione o comportamento suicidario nel soggetto. Di fatto, rispetto alla presenza di una delle scale il rischio (OR) di ideazione suicidaria è 3,8 volte (IC $95 \%=1,7-8,4$ ), con due scale sale al 5,6 (IC 95\% = 2,7-11,6), con tre scale al 20,5 (IC 95\% = 10,7-39,2). Il rischio legato alla "minaccia di suicidio" (scala TS MMPI) presenta una distribuzione ancora più pesante. In presenza di una scala tale rischio risulta 4,5 volte più elevato (IC $95 \%=2,2-8,8), 17,0$ in presenza $\mathrm{di}$ due scale (IC 95\% = 9,3-30,8), 62,8 per tre scale (IC $95 \%=35,4-111,4)$.

Una verifica più particolareggiata delle affermazioni proposte è stata ottenuta dalla regressione logistica tra ideazione suicidaria e comportamento suicida rispetto alle varie combinazioni assunte dall'interazione delle scale D, Sc e LSE del MMPI nel campione indagato.

I risultati riguardanti l'ideazione suicidaria vengono presentati nella Tabella III.

È interessante notare come il rischio (OR) di ideazione suicidaria assuma valori differenziati a seconda delle scale coinvolte e del loro significato clinico. Di fatto il rischio più elevato di avere idee suicide viene trovato nell'interazione tra bassa autostima (LSE) e stile psicotico (Sc), e tra bassa autostima, stile psicotico e struttura depressiva (D). Un'altra condizione significativa viene evidenziata dal fatto che - pur abitualmente ritenuta la variabile più implicata nell'ideazione suicidaria (e nelle condotte autolesive) - alla sola struttura depressiva (scala D) corrisponde il minor rischio per tale processo psicopatologico, segno che testimonia la multidimensionalità di tale fenomeno. Infine, $\mathrm{i}$ dati mostrano come il concorso di uno stile psicotico tenda a disorganizzare pesantemente l'architettura personologica aumentando il rischio di atteggiamenti mentali di natura suicidaria.

I risultati ottenuti dalla regressione logistica tra la scala Threat Suicide (TS) del MMPI e le diverse combinazioni assunte dalle tre scale analizzate, evidenziano un notevole aumento del rischio suicidario rispetto a specifiche interazioni tra le stesse (Tabella IV).

I dati mettono chiaramente in luce come l'interazione tra stile psicotico (Sc), bassa autostima (LSE) e condizione depressiva (D) accrescano in modo rilevante il rischio della messa in atto di comportamenti autolesivi. Inoltre, i risultati ribadiscono come la presenza di una sola scala positiva e la specifica sintomatologia correlata (es. di tipo depressivo, o lo stile psicotico) contribuiscano minimamente a organizzare l'agire suicidario, rispetto alla contemporanea presenza di queste organizzazioni patologiche.

L'analisi della varianza delle tre scale nei gruppi indagati fornisce ulteriori elementi analitici. Di fatto, i valori (medi) assunti dalla scala $D$ nei gruppi $(\mathrm{PG}=51,7 ; \mathrm{DA}=74,2 ; \mathrm{DD}=84,4 ; \mathrm{DS}=71,3 ; \mathrm{F}$ 
$=238,8 ; \mathrm{p}=0,000000)$, dalla scala Sc $(\mathrm{PG}=46,9$; $\mathrm{DA}=65,5 ; \mathrm{DD}=67,6 ; \mathrm{DS}=61,4-\mathrm{F}=106,4 ; \mathrm{p}$ $=0,000000)$, e dalla scala LSE, indicata dai valori numerici positivi ( $\mathrm{PG}=-28,8$; $\mathrm{DA}=2,0$; $\mathrm{DD}=$ $10,6$; $\mathrm{DS}=-5,4 ; \mathrm{F}=223,5 ; \mathrm{p}=0,000000)$, mostrano la maggiore compromissione psicopatologica che caratterizza i quadri depressivi. La verifica di quanto sostenuto è stata ottenuta attraverso la codificazione binaria delle tre scale $(0=$ valori normali della scala, 1 = valori patologici della scala), e la somma di tali valori per ogni gruppo (somma che può assumere valori tra 0 scale e 3 scale). L'analisi della varianza mostra che nella PG il valore medio è 0,1 scale positive; nei $\mathrm{DA}=1,6$; nei $\mathrm{DD}=$ $2,1$; e nei $D S=1,3$ ( $F=184,8 ; \mathrm{p}=0,000000)$, verificando che i quadri depressivi sono gli unici ad avere una media di due scale positive su tre.

Infine, un'ulteriore testimonianza statistica della maggiore disorganizzazione psicopatologica dei quadri depressivi, quindi della loro maggiore vulnerabilità alla suicidalità, è stata ottenuta attraverso alcune analisi statistiche. La regressione lineare tra la somma del valore numerico delle tre scale (D + Sc + LSE) e il valore numerico della scala Threat Suicide del MMPI è risultata molto significativa $(\mathrm{r}=$ 0,$8364 ; p=0,000000$ ), testimonianza che le due variabili misurano una stessa dimensione.

L'analisi della varianza tra la somma del valore numerico delle tre scale (D + Sc + LSE: valore normale $\leq 210$ punti) nei gruppi indagati ha evidenziato, oltre a una significativa differenza tra gli stessi $(\mathrm{F}=$ 360,$4 ; p=0,000000$ ), il più elevato punteggio ottenuto dai depressi (PG = 145,2; $\mathrm{DA}=213,0$; DD $=230,7$; DS $=198,9$ ). L'analisi della varianza della somma citata rispetto all'ideazione suicidaria $(0=$ assenza, 1 = presenza) ha messo in luce che all'assenza di tale processo ideativo corrisponde un valore numerico di 203,1 punti; mentre in sua presenza tale valore sale a 252,5 $(F=286,6 ; p=0,000000)$.

\section{DISCUSSIONE E CONCLUSIONI}

La presente ricerca ha messo in luce come il fenomeno suicidario - nella sua manifestazione interna (ideazione) o obiettiva (minaccia) - sia legato a una dinamica interattiva tra una serie di variabili psicopatologiche che la letteratura e il lavoro hanno rintracciato nelle scale Sc, D e LSE del MMPI (e nel significato clinico ad esse correlato), scale che hanno dimostrato un'ottima consistenza interna (Cronbachs Alpha $=0,92$ ).

Di fatto, queste variabili descrivono una serie di tratti psicopatologici che la ricerca ha evidenziato come intimamente connessi con il fenomeno studiato. La scala Sc (schizofrenia) ha identificato un soggetto caratterizzato da uno "stile psicotico" di approccio con la realtà interiore e quella ambientale. Questa modalità psico-comportamentale di natura patologica si esprime nel ritiro dentro sé, un negativo contatto con la realtà, la produzione di pensieri poco comuni, inadeguati e difficilmente comunicabili, esperienze bizzarre e insolite, non raramente una chiusura di tipo autistico, unitamente a un'elevata propensione per il rischio e una marcata anestesia emozionale, condizioni personologiche che - tra altre conseguenze - non permettendo al soggetto di avvertire sentimenti di paura verso la morte, aumentano la probabilità di acting-out autolesivi.

La scala D ha disegnato un soggetto dall'umore depresso, astenico e sfiduciato, caratterizzato da povertà ideativa, bradipsichismo, ostilità verso sé e gli altri, polarizzazioni pessimistiche del pensiero, idee suicidarie e di morte, unica modalità a disposizione del paziente per tacitare definitivamente la negativa e persecutoria immagine di sé (quella che i pazienti descrivono come la "vocina che mi chiede cosa ci sto a fare al mondo! Mi elenca tutti i miei fallimenti! Mi spinge a essere spietato con me stesso/a!»).

Non casualmente la scala LSE ha proposto un individuo con un'elevata disistima, un senso fallimentare legato alla propria dimensione esistenziale, organizzata sulla percezione di mancanza di valore, su profondi sentimenti di incapacità, su un pervasivo, globale e negativo vissuto di sé.

Nelle risultanze dell'indagine, i pensieri suicidari e la più pericolosa messa in atto degli stessi paiono così strutturarsi all'interno di un terreno dinamico multifattoriale, dove l'incontro tra i pesanti aspetti psicopatologici descritti sgretola letteralmente le precarie difese del paziente, producendo un collasso psichico della sua (fragile) personalità. Nella prospettiva delineata la suicidalità si configura in un modo particolare, proponendosi come un fenomeno dinamico che - pur permeando tutti i quadri clinici (valenza qualitativa) - assume una pesante consistenza (valenza quantitativa) nei disturbi depressivi. Il maggiore coinvolgimento epidemiologico evidenziato dalla patologia affettiva negli atti suicidari troverebbe nel lavoro una spiegazione plausibile (pur necessitante di ulteriori approfondimenti). Di fatto, la profonda compromissione dell'autostima, la severa sintomatologia depressiva, e la presenza di psicoticismo, differenziano statisticamente i quadri depressivi da quelli somatoformi e d'ansia, assegnando ai primi un particolare profilo psicopatologico.

I dati ottenuti portano così a valutare che i quadri depressivi evidenziano un maggiore "peso psicopatologico" delle tre variabili indagate, situazione che - oltre a rivelarsi di difficoltosa gestione - sollecita nel paziente depresso un arcaico sistema difensivo che è possibile rappresentare attraverso l'equazione psicodinamica "eliminazione del peso psicopatologico = eliminazione della propria persona". Il 
profondo livello di disorganizzazione personologica che caratterizza i quadri depressivi, di conseguenza, aumenta la probabilità di pensieri e comportamenti autolesivi, rendendo questa categoria psicopatologica maggiormente permeabile a tali fenomeni.

\section{DISCLOSURE}

L'Autore dichiara di non avere conflitti di interesse di natura finanziaria.

\section{BIBLIOGRAFIA}

1. Black DW, Warrack G, Winokur G. The lowa record-linkage study. I. Suicides and accidental deaths among psychiatric patients. Arch Gen Psychiatry 1988; 42: 71-5

2. Oquendo MA, Galfalvy H, Russo S, Ellis PS, Grunebaum MF, Burke A et al. Prospective study of clinical predictors of suicidal acts after a major depressive episode in patients with major depressive disorder or bipolar disorder. Am J Psychiatry 2004;161: 1433-41

3. Goldstein RB, Black DW, Nasrallah A, Winokur G. The prediction of suicide: sensitivity, specificity and predictive value of a multivariate model applied to suicide among 1906 patients with affective disorders. Arch Gen Psychiatry 1991; 48: 418-22

4. Sharma R, Markar HR. Mortality in affective disorder. J Affect Disord 1994; 31: 91-6

5. Nordström P, Asberg M, Aberg-Wistedt A, Nordin C. Attempted suicide predicts suicide risk in mood disorder. Acta Psychiatr Scand 1995; 92: 345-50

6. Blair-West GW, Mellsop GW. Down-rating lifetime suicide risk in major depression. ActaPsychiatr Scand 1997; 9: 259-63

7. Steer RA, Rissmiller DJ, Ranieri WF, Beck AT. Dimensions of suicidal ideation in psychiatric inpatients. Behav Res Ther 1993; 31: 229-36

8. Brown GK, Beck AT, Steer RA, Grisham JR. Risk factors for suicide in psychiatric outpatients: a 20-year prospective study. J Consult Clin Psychol 2000; 68: 371-7

9. Kontaxakis V, Havaki-Kontaxaki B, Margariti M, Stamouli S, Kollias C, Christodoulou G. Suicidal ideation in inpatients with acute schizophrenia. Can J Psychiatry 2004; 49: 476-9

10. Terra JL. Suicide risk and depression. Rev Prat 2008; 58: 385-8

11. Palmer CJ Jr. Suicide attempt history, self-esteem, and suicide risk in a sample of 116 depressed voluntary inpatients. Psychol Rep 2004; 95: 1092-4

12. Williams JM, Crane C, Barnhofer T, Van der Does AJ, Segal ZV. Recurrence of suicidal ideation across depressive episodes. J Affect Disord 2006; 91: 189-94

13. Wild LG, Flisher AJ, Lombard C. Suicidal ideation and attempts in adolescents: associations with depression and six domains of self-esteem.J Adolesc 2004; 27: 611-24

14. Franck E, De Raedt R, Dereu M, Van den Abbeele D. Implicit and explicit self-esteem in currently depressed individuals with and without suicidal ideation. J Behav Ther Exp Psychiatry 2007; 38: 75-85

15. Pantusa MF, Berardi M, Paparo S, Scornaienchi C. Differenze di genere e sintomatologia depressiva in adolescenza: relazioni tra autostima, sintomi depressivi e ideazione suicidaria. Giorn Ital Psicopat 2006; 12: 407-14

16. Cohen LJ, Test MA, Brown RL. Suicide and schizophrenia: data from a prospective community treatment study. Am J Psychiatry 1990; 147: 602-7

17. Goldstein G, Haas GL, Pakrashi M, Novero AM, Luther JF. The cycle of schizoaffective disorder, cognitive ability, alcoholism, and suicidality. Suicide Life Threat Behav 2006; 36: 35-43

18. Mosticoni R, Chiari G. Una descrizione obiettiva della personalità: il Minnesota Multiphasic Personality Inventory (MMPI). Firenze: Organizzazioni Speciali, 1979

19. Baumgardner AH. To know oneself is to like oneself: Self-certainty and self-affect.J Pers Soc Psychol 1990; 58: 1062-72

20. Branden N. The six pillars of Self-esteem. New York: Bantam Books, 1994

21. Pfeffer CR. Suicide prevention: current efficacy and future promise. Ann NY Acad Sci 1986; 487: 341-50

22. Murphy GE, Cooper-Patrick L, Crum RM, Ford DE. Identifying suicidal ideation in general medical patients. JAMA 1994; 272: 1757-62

23. McHolm AE, MacMillan HL, Jamieson E. The relationship between childhood physical abuse and suicidality among depressed women: results from a community sample. Am J Psychiatry 2003; 160: 933-8

24. Infrasca R. Stile genitoriale e ideazione suicidaria nel paziente psichiatrico. Giorn Ital Psicopat 2009; 15: 186-92

25. Farberow NL, Devries AG. An item differentiation analysis of MMPIs of suicidal neuropsychiatric hospital patients. Psychol Rep 1967; 20: 607-17

\section{CORRESPONDING AUTHOR}

Dott. Roberto Infrasca. Dipartimento di Psichiatria - Asl 5. Via Zara 2, 19122 La Spezia. Tel: 0187-534977

E-mail: roberto.infrasca@asl5.liguria.it 